\title{
On determinants and inverses of some triband Toeplitz ma- trices with permuted columns
}

\author{
Pingyun $\mathrm{Li}^{\mathrm{a}}$, Zhaolin Jiang ${ }^{\mathrm{a}, *}$, Yanpeng Zheng ${ }^{\mathrm{b}, *}$ \\ a School of Mathematics and Statistics, Linyi University, Linyi 276000, China. \\ ${ }^{b}$ School of Automation and Electrical Engineering, Linyi University, Linyi 276000, China.
}

\begin{abstract}
In this paper, we study the triband Toeplitz and Hankel matrices with permuted columns. We obtain expressions for the determinants and the inverses of the triband Toeplitz and Hankel matrices with permuted columns by the Sherman-MorrisonWoodbury formula, where the Pell numbers play an essential role.
\end{abstract}

Keywords: Determinant, inverse, Laplace theorem, Pell number, triband Toeplitz matrices with permuted columns, Sherman-Morrison-Woodbury.

2010 MSC: 15A09, 15A15, 15B05, 65F40.

(C)2020 All rights reserved.

\section{Introduction}

In this paper, we study the determinants and inverses of triband Toeplitz matrices with permuted columns and triband Hankel matrices with permuted columns, respectively. The matrix $A=\left(a_{i, j}\right)_{i, j=1}^{n}$ is called a triband Toeplitz matrices with permuted columns, whose entries are as follows

$$
a_{i, j}= \begin{cases}\delta_{i}, & 1 \leqslant i \leqslant n, j=1 \\ \epsilon_{i}, & 1 \leqslant i \leqslant n, j=n \\ -v, & i=j+2,2 \leqslant j \leqslant n-1, \\ -2 v, & i=j+1,2 \leqslant j \leqslant n-1, \\ v, & 2 \leqslant i=j \leqslant n-1 \\ 0, & \text { otherwise, }\end{cases}
$$

where $\epsilon_{1}, \cdots \epsilon_{n}, \delta_{1}, \cdots \delta_{n}$ and $v$ are complex numbers with $v \neq 0$. More explicitly,

\footnotetext{
*Corresponding authors

Email address: jzh1208@sina.com (Zhaolin Jiang)
}

doi: $10.22436 /$ jmcs.020.03.02

Received: 2019-08-13 Revised: 2019-10-11 Accepted: 2019-10-28 


$$
A=\left(\begin{array}{ccccccc}
\delta_{1} & 0 & \cdots & \cdots & \cdots & 0 & \epsilon_{1} \\
\delta_{2} & v & 0 & & & \vdots & \epsilon_{2} \\
\delta_{3} & -2 v & v & \ddots & & \vdots & \epsilon_{3} \\
\delta_{4} & -v & -2 v & v & \ddots & \vdots & \vdots \\
\vdots & 0 & -v & -2 v & \ddots & 0 & \vdots \\
\vdots & \vdots & \ddots & \ddots & \ddots & v & \epsilon_{n-1} \\
\delta_{n} & 0 & \cdots & 0 & -v & -2 v & \epsilon_{n}
\end{array}\right)_{n \times n}
$$

Let $\hat{I}_{n}$ be the $n \times n$ "reverse unit matrix", which has ones along the secondary diagonal and zeros elsewhere. That is,

$$
\hat{\mathrm{I}}_{\mathrm{n}}=\left(\begin{array}{cccc}
0 & \cdots & 0 & 1 \\
\vdots & . \cdot & . & 0 \\
0 & . \cdot & . & \vdots \\
1 & 0 & \cdots & 0
\end{array}\right)_{\mathrm{n} \times \mathrm{n}}
$$

Let $A$ be defined as in (1.1). A matrix of the form $B:=\hat{I}_{n} A$ is called a triband Hankel matrices with permuted columns of Type I. A matrix of the form $C:=A \hat{I}_{n}$ is called a triband Hankel matrices with permuted columns of Type II. In this case, we say B and C are induced by $A$. That is,

$$
\mathrm{B}=\left(\begin{array}{ccccccc}
\delta_{\mathrm{n}} & 0 & \cdots & 0 & -v & -2 v & \epsilon_{\mathrm{n}} \\
\delta_{n-1} & \vdots & . \cdot & -v & -2 v & v & \epsilon_{\mathrm{n}-1} \\
\vdots & 0 & . \cdot & . \cdot & . \cdot & 0 & \epsilon_{\mathrm{n}-2} \\
\vdots & -v & . \cdot & v & . \cdot & \vdots & \vdots \\
\delta_{3} & -2 v & . \cdot & 0 & & \vdots & \vdots \\
\delta_{2} & v & 0 & & & 0 & \epsilon_{2} \\
\delta_{1} & 0 & \cdots & \ldots & \ldots & 0 & \epsilon_{1}
\end{array}\right)_{\mathrm{n} \times \mathrm{n}}
$$

and

$$
C=\left(\begin{array}{ccccccc}
\epsilon_{1} & 0 & \ldots & \ldots & \ldots & 0 & \delta_{1} \\
\epsilon_{2} & 0 & & & 0 & v & \delta_{2} \\
\epsilon_{3} & 0 & & . \cdot & . & -2 v & \delta_{3} \\
\vdots & \vdots & . \cdot & v & . \cdot & -v & \vdots \\
\epsilon_{n-1} & 0 & . \cdot & . \cdot & . \cdot & 0 & \delta_{n-2} \\
\epsilon_{n-1} & v & -2 v & -v & . \cdot & \vdots & \delta_{n-1} \\
\epsilon_{n} & -2 v & -v & 0 & \ldots & 0 & \delta_{n}
\end{array}\right)_{n \times n}
$$

Many special banded matrices, such as Toeplitz banded matrix, symmetric Toeplitz banded matrix, especially tridiagonal Toeplitz matrix, can be seen in $[4,5,8,9,12]$. The inverse of Toeplitz banded matrix has been studied by many scholars. For example, Roebuck and Barnett [14] made some good conclusions about Toeplitz matrix. Yamamoto and Ikebe [16] studied the inverse matrix of general band matrix, including the inverse matrix of special Toeplitz band matrix, while the concrete formula of the inverse matrix of Toeplitz band matrix is deduced in [15]. Besides, Bareiss [2] proposed a recursive method for 
solving Toeplitz equations. Both $[1,11]$ gave a method for calculating the inverse matrix of symmetric Toeplitz strip matrix. Especially, two special cases of two symmetric Toeplitz band matrices are studied in $[7,13]$.

Now we introduce a Pell number sequence [3, 10,17-19] that plays a very important role in our main results. The Pell number $P_{n}$ satisfies the following recurrence:

$$
P_{n}=2 P_{n-1}+P_{n-2}, \text { where } P_{0}=0, P_{1}=1, n \geqslant 2 \text {. }
$$

It could be verified that

$$
\begin{gathered}
\sum_{i=1}^{n} i P_{i}=\frac{3 n-2}{2} P_{n}+\frac{n-1}{2} P_{n-1}+\frac{1}{2}, \\
\sum_{i=1}^{n} P_{n+1-i} a^{i}=\frac{a P_{n}+a^{2} P_{n+1}-a^{n+2}}{1+2 a-a^{2}}(a \neq 1 \pm \sqrt{2}) .
\end{gathered}
$$

\section{Determinants and inverses}

In this section, we derive explicit formulas for the determinants and inverses of triband Toeplitz matrices with permuted columns.

Theorem 2.1. Let $A=\left(a_{i, j}\right)_{i, j=1}^{n}(n \geqslant 3)$ be given as in (1.1). Then

$$
\operatorname{det} A=\sum_{m=1}^{n-1} P_{n-m}\left(\delta_{1} v^{n-2} \epsilon_{m+1}+(-1)^{n+1} \epsilon_{1} v^{n-2} \delta_{m+1}\right),
$$

where $P_{i}(i=n-m)$ is the $i^{\text {th }}$ Pell number.

Proof. Expanding matrix $A=\left(a_{i, j}\right)_{i, j=1}^{n}(n \geqslant 3)$ by the first row, we get

$$
\operatorname{det} A=\delta_{1} \operatorname{det} A_{1}+(-1)^{n+1} \epsilon_{1} \operatorname{det} A_{2} \text {, }
$$

where

and

$$
A_{1}=\left(\begin{array}{cccccc}
v & 0 & \cdots & \cdots & \cdots & \epsilon_{2} \\
-2 v & v & \ddots & & \vdots & \epsilon_{3} \\
-v & -2 v & v & \ddots & \vdots & \vdots \\
0 & -v & -2 v & \ddots & 0 & \vdots \\
\vdots & \ddots & \ddots & \ddots & v & \epsilon_{n-1} \\
0 & \cdots & 0 & -v & -2 v & \epsilon_{n}
\end{array}\right)_{(n-1) \times(n-1)}
$$

$$
A_{2}=\left(\begin{array}{cccccc}
\delta_{2} & v & 0 & \cdots & \cdots & 0 \\
\delta_{3} & -2 v & v & \ddots & & \vdots \\
\delta_{4} & -v & -2 v & v & \ddots & \vdots \\
\vdots & 0 & -v & -2 v & \ddots & 0 \\
\vdots & \vdots & \ddots & \ddots & \ddots & v \\
\delta_{n} & 0 & \cdots & 0 & -v & -2 v
\end{array}\right)_{(n-1) \times(n-1)}
$$


By performing a series of elementary transformations on $A_{1}$, we obtain the matrix

$$
\mathrm{B}_{1}=\left(\begin{array}{cccccc}
v & 0 & \cdots & \cdots & \cdots & \epsilon_{2} \\
0 & v & \ddots & & \vdots & \epsilon_{3}+2 \epsilon_{2} \\
0 & 0 & v & \ddots & \vdots & \epsilon_{4}+2 \epsilon_{3}+5 \epsilon_{2} \\
0 & 0 & 0 & \ddots & 0 & \vdots \\
\vdots & \ddots & \ddots & \ddots & v & \sum_{m=1}^{n-2} P_{n-m} \epsilon_{m+1} \\
0 & \cdots & 0 & 0 & 0 & \sum_{m=1}^{n-1} P_{n-m} \epsilon_{m+1}
\end{array}\right)_{(n-1) \times(n-1)}
$$

Calculating the determinant of matrix $\mathrm{B}_{1}$,

$$
\operatorname{det} B_{1}=v^{n-2} \sum_{m=1}^{n-1} P_{n-m} \epsilon_{m+1} .
$$

Since all elementary transformation preserve the determinants, we get

$$
\operatorname{det} A_{1}=\operatorname{det} B_{1}=v^{n-2} \sum_{m=1}^{n-1} P_{n-m} \epsilon_{m+1} .
$$

Similarly, by performing a series of elementary transformations on $A_{2}$, we obtain the matrix

$$
\mathrm{B}_{2}=\left(\begin{array}{cccccc}
\delta_{2} & v & 0 & \cdots & \cdots & 0 \\
\delta_{3}+2 \delta_{2} & 0 & v & \ddots & & \vdots \\
\delta_{4}+2 \delta_{3}+5 \delta_{2} & 0 & 0 & v & \ddots & 0 \\
\vdots & 0 & 0 & 0 & \ddots & 0 \\
\vdots & \vdots & \ddots & \ddots & \ddots & v \\
\sum_{m=1}^{n-1} P_{n-m} \delta_{m+1} & 0 & \cdots & 0 & 0 & 0
\end{array}\right)_{(n-1) \times(n-1)}
$$

We also have

$$
\operatorname{det} A_{2}=\operatorname{det} B_{2}=v^{n-2} \sum_{m=1}^{n-1} P_{n-m} \delta_{m+1}
$$

Based on (2.1), (2.3), and (2.4), we get

$$
\operatorname{det} A=\sum_{m=1}^{n-1} P_{n-m}\left(\delta_{1} v^{n-2} \epsilon_{m+1}+(-1)^{n+1} \epsilon_{1} v^{n-2} \delta_{m+1}\right) .
$$

Corollary 2.2. Let $\delta_{j}=\delta+(n+1-j) d_{1}$ and $\epsilon_{j}=\epsilon+(n+1-j) d_{2}, j=1,2, \ldots, n$ in $(1.1)$, where $\delta, \epsilon, d_{1}$ and $\mathrm{d}_{2}$ are nonzero complex numbers. Then

$$
\begin{aligned}
\operatorname{det} A= & v^{n-2}\left(\delta+n d_{1}\right)\left(\epsilon \frac{P_{n-1}+P_{n}-1}{2}+d_{2} \frac{(n-2) P_{n}+(n-1) P_{n-1}+1}{2}\right) \\
& +(-1)^{n+1} v^{n-2}\left(\epsilon+n d_{2}\right)\left(\delta \frac{P_{n-1}+P_{n}-1}{2}+d_{1} \frac{(n-2) P_{n}+(n-1) P_{n-1}+1}{2}\right) .
\end{aligned}
$$


Corollary 2.3. Let $\delta_{j}=\delta q_{1}^{j}$ and $\epsilon_{j}=\epsilon q_{2}^{j}, j=1,2, \ldots, n$ in $(1.1)$, where $\delta, \epsilon, q_{1}$ and $q_{2}$ are nonzero complex numbers. Then

$$
\operatorname{det} A=\delta \in q_{1}\left(\frac{q_{2} P_{n}+q_{2}^{2} P_{n+1}-q_{2}^{n+2}}{1+2 q_{2}-q_{2}^{2}}-P_{n} q_{2}\right)-\delta \epsilon q_{2}\left(\frac{q_{1} P_{n}+q_{1}^{2} P_{n+1}-q_{1}^{n+2}}{1+2 q_{1}-q_{1}^{2}}-P_{n} q_{1}\right) .
$$

Theorem 2.4. Let $A=\left(a_{i, j}\right)_{i, j=1}^{n}(n \geqslant 3)$ be given as in (1.1) and assume $A$ to be nonsingular. Then $A^{-1}=$ $\left(\breve{a}_{i, j}\right)_{i, j=1}^{n}$, where

$$
\begin{aligned}
& \breve{a}_{i, j}= \begin{cases}\frac{\sum_{m=2}^{n} P_{n+1-m} \epsilon_{m}}{a}, & i=1, j=1, \\
-\frac{\sum_{m=2}^{n} P_{n+1-m} \delta_{m}}{a}, & i=n, j=1, \\
-\frac{\sum_{m=2}^{n} P_{n+1-m}\left(\epsilon_{m} \sum_{h=2}^{i} P_{i+1-h} \delta_{h}-\delta_{m} \sum_{h=2}^{i} P_{i+1-h} \epsilon_{h}\right)}{v a}, & 2 \leqslant i \leqslant n-1, j=1, \\
-\frac{P_{n+1-j} \epsilon_{1}}{a}, & i=1,2 \leqslant j \leqslant n, \\
\frac{P_{n+1-j} \delta_{1}}{a}, j & i=n, 2 \leqslant j \leqslant 2, \\
-\frac{P_{n+1-j} \sum_{h=2}^{i} P_{i+1-h}\left(\epsilon_{h} \delta_{1}-\delta_{h} \epsilon_{1}\right)}{v a}, & 2 \leqslant i \leqslant n-1,2 \leqslant j \leqslant n, i<j, \\
\frac{a P_{i-j+1}-P_{n+1-j} \sum_{h=2}^{i} P_{i+1-h}\left(\epsilon_{h} \delta_{1}-\delta_{h} \epsilon_{1}\right)}{v a}, & 2 \leqslant i \leqslant n-1,2 \leqslant j \leqslant n, i \geqslant j,\end{cases} \\
& a=\sum_{m=1}^{n} P_{n+1-m}\left(\delta_{1} \epsilon_{m}-\epsilon_{1} \delta_{m}\right)
\end{aligned}
$$

and $\mathrm{P}_{\mathrm{i}}$ is the $\mathrm{i}^{\text {th }}$ Pell number.

Proof. We decompose $A$ as follows

$$
A=v \Delta+\mathrm{LE},
$$

where

$$
\Delta=\left(\begin{array}{cccccc}
1 & 0 & \cdots & \cdots & \cdots & 0 \\
-2 & 1 & \ddots & & & 0 \\
-1 & -2 & 1 & \ddots & & \vdots \\
0 & -1 & -2 & 1 & \ddots & \vdots \\
\vdots & \ddots & \ddots & \ddots & \ddots & 0 \\
0 & \cdots & 0 & -1 & -2 & 1
\end{array}\right)_{n \times n} \quad, \quad L=\left(\begin{array}{cc}
\delta_{1}-v & \epsilon_{1} \\
\delta_{2}+2 v & \epsilon_{2} \\
\delta_{3}+v & \epsilon_{3} \\
\delta_{4} & \epsilon_{4} \\
\vdots & \vdots \\
\delta_{n-1} & \epsilon_{n-1} \\
\delta_{n} & \epsilon_{n}-v
\end{array}\right)_{n \times 2}
$$

and

$$
E=\left(\begin{array}{llllll}
1 & 0 & \cdots & \cdots & 0 & 0 \\
0 & 0 & \cdots & \cdots & 0 & 1
\end{array}\right)_{2 \times n}
$$

By some computation, it is not difficult to find that the inverse of $\Delta$ is

$$
\Delta^{-1}=\left(\begin{array}{ccccc}
\mathrm{P}_{1} & 0 & \cdots & \cdots & 0 \\
\mathrm{P}_{2} & \mathrm{P}_{1} & \ddots & & \vdots \\
\vdots & \ddots & \ddots & \ddots & \vdots \\
\mathrm{P}_{n-1} & \ddots & \ddots & \mathrm{P}_{1} & 0 \\
\mathrm{P}_{\mathrm{n}} & \mathrm{P}_{\mathrm{n}-1} & \cdots & \mathrm{P}_{2} & \mathrm{P}_{1}
\end{array}\right)_{n \times n}
$$

where $P_{i}$ is the $i^{\text {th }}$ Pell number. 
Applying the Sherman-Morrison-Woodbury formula (see, e.g., [6, p.50]) to (2.7), we get

$$
\mathrm{A}^{-1}=(v \Delta+\mathrm{LE})^{-1}=\frac{1}{v} \Delta^{-1}-\frac{1}{v^{2}} \Delta^{-1} \mathrm{~L}\left(\mathrm{I}+\frac{1}{v} \mathrm{E} \Delta^{-1} \mathrm{~L}\right)^{-1} \mathrm{E} \Delta^{-1} .
$$

Now we compute each component on the right side of (2.8). Multiplying $\Delta^{-1}$ by $E$ from left, we get

$$
\mathrm{E} \Delta^{-1}=\left(\begin{array}{ccccc}
1 & 0 & \cdots & \cdots & 0 \\
\mathrm{P}_{\mathrm{n}} & \mathrm{P}_{\mathrm{n}-1} & \cdots & \mathrm{P}_{2} & \mathrm{P}_{1}
\end{array}\right)_{2 \times \mathrm{n}} .
$$

Consider multiplying $\mathrm{E} \Delta^{-1}$ by $\mathrm{L}$ from right yields, we get

$$
E \Delta^{-1} L=\left(\begin{array}{cc}
\delta_{1}-v & \epsilon_{1} \\
\sum_{m=1}^{n} P_{n+1-m} \delta_{m} & \sum_{m=1}^{n} P_{n+1-m} \epsilon_{m}-P_{1} v
\end{array}\right)_{2 \times 2}
$$

Then multiplying (2.9) by $\frac{1}{v}$ from the left and further adding I, we have

$$
\mathrm{I}+\frac{1}{v} \mathrm{E} \Delta^{-1} \mathrm{~L}=\frac{1}{v}\left(\sum_{m=1}^{n} \mathrm{P}_{\mathrm{n}+1-\mathrm{m}} \delta_{\mathrm{m}} \sum_{\mathrm{m}=1}^{\mathrm{n}} \mathrm{P}_{\mathrm{n}+1-\mathrm{m}} \epsilon_{\mathrm{m}}\right)_{2 \times 2} .
$$

Computing the inverse of the matrices on both sides of (2.10), we obtain

$$
\left(\mathrm{I}+\frac{1}{v} \mathrm{E} \Delta^{-1} \mathrm{~L}\right)^{-1}=\frac{1}{v \mathrm{~d}}\left(\begin{array}{cc}
\sum_{m=1}^{\mathrm{n}} \mathrm{P}_{\mathrm{n}+1-\mathrm{m}} \epsilon_{\mathrm{m}} & -\epsilon_{1} \\
-\sum_{\mathrm{m}=1}^{\mathrm{n}} \mathrm{P}_{\mathrm{n}+1-\mathrm{m}} \delta_{\mathrm{m}} & \delta_{1}
\end{array}\right)_{2 \times 2},
$$

where

$$
\mathrm{d}=\frac{\delta_{1} \sum_{\mathrm{m}=1}^{\mathrm{n}} \mathrm{P}_{\mathrm{n}+1-\mathrm{m}} \epsilon_{\mathrm{m}}-\epsilon_{1} \sum_{\mathrm{m}=1}^{\mathrm{n}} \mathrm{P}_{\mathrm{n}+1-\mathrm{m}} \delta_{\mathrm{m}}}{v^{2}} .
$$

Multiplying $\Delta^{-1}$ by L from the right, we get

$$
\Delta^{-1} L=\left(\begin{array}{cc}
\delta_{1}-v & \epsilon_{1} \\
\sum_{m=1}^{2} P_{3-m} \delta_{m} & \sum_{m=1}^{2} P_{3-m} \epsilon_{m} \\
\vdots & \vdots \\
\sum_{m=1}^{n-1} P_{n-m} \delta_{m} & \sum_{m=1}^{n-1} P_{n-m} \epsilon_{m} \\
\sum_{m=1}^{n=1} P_{n+1-m} \delta_{m} & \sum_{m=1}^{n} P_{n+1-m} \epsilon_{m}-v
\end{array}\right)_{n \times 2}
$$

Multiplying $\left(\mathrm{I}+\frac{1}{v} \mathrm{E} \Delta^{-1} \mathrm{~L}\right)^{-1}$ by $\Delta^{-1} \mathrm{~L}$ from the left, yields

$$
\Delta^{-1} \mathrm{~L}\left(\mathrm{I}+\frac{1}{v} \mathrm{E} \Delta^{-1} \mathrm{~L}\right)^{-1}=\left(\begin{array}{cc}
\alpha_{1} & \beta_{1} \\
\alpha_{2} & \beta_{2} \\
\vdots & \vdots \\
\alpha_{n} & \beta_{n}
\end{array}\right)_{n \times 2}
$$

where

$$
\alpha_{i}= \begin{cases}v-\frac{\sum_{m=1}^{n} P_{n+1-m} \epsilon_{m}}{d}, & i=1, \\ P_{i} v+\frac{\sum_{h=2}^{i} P_{i+1-h}\left(\delta_{h} \sum_{m=1}^{n} P_{n+1-m} \epsilon_{m}-\epsilon_{h} \sum_{m=1}^{n} P_{n+1-m} \delta_{m}\right)}{v d}, & 2 \leqslant i \leqslant n-1, \\ \frac{\sum_{m=1}^{n} P_{n+1-m} \delta_{m}}{d}, & i=n,\end{cases}
$$


and

$$
\beta_{i}= \begin{cases}\frac{\epsilon_{1}}{d}, & i=1, \\ \sum_{h=2}^{i} P_{i+1-h}\left(\epsilon_{h} \delta_{1}-\delta_{h} \epsilon_{1}\right) & \\ v-\frac{\delta_{1}}{d}, v & 2 \leqslant i \leqslant n-1, \\ & i=n .\end{cases}
$$

Multiplying the previous formula $\left(\mathrm{I}+\frac{1}{v} \mathrm{E} \Delta^{-1} \mathrm{~L}\right)^{-1}$ by $\Delta^{-1} \mathrm{~L}$ from the left and by $\mathrm{E} \Delta^{-1}$ from the right, respectively, yields

$$
\mathrm{G}=\Delta^{-1} \mathrm{~L}\left(\mathrm{I}+\frac{1}{v} \mathrm{E} \Delta^{-1} \mathrm{~L}\right)^{-1} \mathrm{E} \Delta^{-1}=\left(g_{i j}\right)_{i, j=1}^{n}
$$

where

$$
g_{i j}= \begin{cases}v-\frac{v^{2} \sum_{m=2}^{n} P_{n+1-m} \epsilon_{m}}{a}, & i=1, j=1, \\ P_{n} v+v^{2} \frac{\sum_{m=2}^{n} p_{n+1-m} \delta_{m}}{a}, & i=n, j=1, \\ \frac{v^{2} P_{n+1-j} \epsilon_{1}}{a} \sum_{\sum_{m=2}^{n} P_{n+1-m}\left(\epsilon_{m} \sum_{h=2}^{i} P_{i+1-h} \delta_{h}-\delta_{m} \sum_{h=2}^{i} P_{i+1-h} \epsilon_{h}\right)}^{a}, & i=1,2 \leqslant j \leqslant n, \\ P_{i} v+v \frac{m \leqslant n-1, j=1,}{P_{n+1-j} \sum_{h=2}^{i} P_{i+1-h}\left(\epsilon_{h} \delta_{1}-\delta_{h} \epsilon_{1}\right)}, & 2 \leqslant i \leqslant n-1,2 \leqslant j \leqslant n, \\ v \frac{a}{P_{n+1-j} v-v^{2} \frac{P_{n+1-j} \delta_{1}}{a},}, & i=n, 2 \leqslant j \leqslant n,\end{cases}
$$

and

$$
a=\sum_{m=1}^{n} P_{n+1-m}\left(\delta_{1} \epsilon_{m}-\epsilon_{1} \delta_{m}\right) .
$$

From (2.8) and (2.11), we have

$$
\left(\breve{a}_{i, j}\right)_{i, j=1}^{n}=\frac{1}{v} \Delta^{-1}-\frac{1}{v^{2}}\left(g_{i j}\right)_{i, j=1}^{n}
$$

where

$$
\breve{a}_{i, j}= \begin{cases}\frac{\sum_{m=2}^{n} P_{n+1-m} \epsilon_{m}}{a}, & i=1, j=1, \\ -\frac{\sum_{m=2}^{n} P_{n+1-m} \delta_{m}}{a}, \quad & i=n, j=1, \\ -\frac{\sum_{m=2}^{n} P_{n+1-m}\left(\epsilon_{m} \sum_{h=2}^{i} P_{i+1-h} \delta_{h}-\delta_{m} \sum_{h=2}^{i} P_{i+1-h} \epsilon_{h}\right)}{v a}, & i \leqslant i \leqslant n-1, j=1, \\ -\frac{P_{n+1-j} \epsilon_{1}}{a}, & i=n, 2 \leqslant j \leqslant n, \\ \frac{P_{n+1-j} \delta_{1}}{a},{ }^{i} & 2 \leqslant i \leqslant n-1,2 \leqslant j \leqslant n, i<j, \\ -\frac{P_{n+1-j} \sum_{h=2}^{i} P_{i+1-h}\left(\epsilon_{h} \delta_{1}-\delta_{h} \epsilon_{1}\right)}{\nu a}, & 2 \leqslant i \leqslant n-1,2 \leqslant j \leqslant n, i \geqslant j .\end{cases}
$$


Corollary 2.5. Let $\delta_{j}=\delta+(n+1-j) d_{1}$ and $\epsilon_{j}=\epsilon+(n+1-j) d_{2}, j=1,2, \ldots, n$ in (1.1), where $\delta, \epsilon, d_{1}$ and $\mathrm{d}_{2}$ are nonzero complex numbers. Then $\mathrm{A}^{-1}=\left(\dot{\mathrm{a}}_{i, j}\right)_{i, j=1}^{n}$, where

$$
\begin{aligned}
\dot{a}_{i, j} & = \begin{cases}\frac{P_{n} \epsilon+(n-2) P_{n} d_{2}+P_{n-1} \epsilon+(n-1) P_{n-1} d_{2}+d_{2}-\epsilon}{2 c}, & i=1, j=1, \\
-\frac{P_{n} \delta+(n-2) P_{n} d_{1}+P_{n-1} \delta+(n-1) P_{n-1} d_{1}+d_{1}-\delta}{2 c}, & i=n, j=1, \\
\frac{\left(\delta d_{2}-\epsilon d_{1}\right) b}{4 v c}, & 2 \leqslant i \leqslant n-1, j=1, \\
-\frac{P_{n+1-j}\left(\epsilon+n d_{2}\right)}{c}, & i=1,2 \leqslant j \leqslant n, \\
\frac{P_{n+1-j}\left(\delta+n d_{1}\right)}{c}, & i=n, 2 \leqslant j \leqslant n, \\
-P_{n+1-j}\left(\epsilon d_{1}-\delta d_{2}\right) \frac{P_{i+1}-i-1}{2 v c}, & 2 \leqslant i \leqslant n-1,2 \leqslant j \leqslant n, i<j, \\
\frac{P_{i-j+1}-P_{n+1-j}\left(\epsilon d_{1}-\delta d_{2}\right) \frac{P_{i+1}-i-1}{2 v c},}{v}, & 2 \leqslant i \leqslant n-1,2 \leqslant j \leqslant n, i \geqslant j,\end{cases} \\
b & =P_{i-1} P_{n}-n P_{i-1}-P_{i} P_{n-1}-(n-1) P_{i}+i P_{n-1}+(i-1) P_{n}+n-i, \\
c & =\left(\delta d_{2}-\epsilon d_{1}\right) \frac{-2 P_{n}-P_{n-1}+n+1}{2},
\end{aligned}
$$

and $\mathrm{P}_{\mathrm{i}}$ is the $\mathrm{i}^{\text {th }}$ Pell number.

Proof. We can obtain this conclusion by using (1.4), (1.5), and Theorem 2.4.

Corollary 2.6. Let $\delta_{j}=\delta q_{1}^{j}$ and $\epsilon_{j}=\epsilon q_{2}^{j}, j=1,2, \ldots, n$ in (1.1), where $\delta, \epsilon, q_{1}$ and $q_{2}$ are nonzero complex numbers. Then $A^{-1}=\left(\ddot{a}_{i, j}\right)_{i, j=1}^{n}$, where

$$
\begin{aligned}
& \ddot{a}_{i, j}= \begin{cases}\frac{\left(q_{2}^{2} P_{n-1}-q_{2}^{n+2}+P_{n} q_{2}^{3}\right) \epsilon}{d+2 q_{2} d-q_{2}^{2} d}, & i=1, j=1, \\
-\frac{\left(q_{1}^{2} P_{n-1}-q_{1}^{n+2}+P_{n} q_{1}^{3}\right) \delta}{d+2 q_{1} d-q_{1}^{2} d}, & i=n, j=1, \\
\frac{\epsilon \delta e}{v d\left(1+2 q_{1}-q_{1}^{2}\right)\left(1+2 q^{2}-q_{2}^{2}\right)}, & 2 \leqslant i \leqslant n-1, j=1, \\
-\frac{P_{n+1-j} \in q_{2}}{d}, & i=1,2 \leqslant j \leqslant n, \\
\frac{P_{n+1-j} \delta q_{1}}{d}, & i=n, 2 \leqslant j \leqslant n, \\
-P_{n+1-j} \delta \epsilon\left(\frac{P_{i-1}+q_{2}^{2} q_{1}+P_{i} q_{2}^{3} q_{1}-q_{2}^{i+2} q_{1}}{v d+2 v d q_{2}-v d q_{2}^{2}}-\right. & \\
\frac{P_{i-1}+q_{1}^{2} q_{2}+P_{i} q_{1}^{3} q_{2}-q_{1}^{i+2} q_{2}}{v d}, & 2 \leqslant i \leqslant n-1,2 \leqslant j \leqslant n, i<j, \\
\frac{P_{i-j+1}-2 v d q_{1}-v d q_{1}^{2}}{d}-P_{n+1-j} \delta \epsilon\left(\frac{P_{i-1}+q_{2}^{2} q_{1}+P_{i} q_{2}^{3} q_{1}-q_{2}^{i+2} q_{1}}{v d+2 v d q_{2}-v d q_{2}^{2}}-\right. & \\
\left.\frac{P_{i-1}+q_{1}^{2} q_{2}+P_{i} q_{1}^{3} q_{2}-q_{1}^{i+2} q_{2}}{v d+2 v d q_{1}-v d q_{1}^{2}}\right), & 2 \leqslant i \leqslant n-1,2 \leqslant j \leqslant n, i \geqslant j,\end{cases} \\
& d=\delta \epsilon\left(\frac{q_{2} q_{1} P_{n}+q_{2}^{2} q_{1} P_{n+1}-q_{1} q_{2}^{n+2}}{1+2 q_{2}-q_{2}^{2}}-\frac{q_{2} q_{1} P_{n}+q_{1}^{2} q_{2} P_{n+1}-q_{2} q_{1}^{n+2}}{1+2 q_{1}-q_{1}^{2}}\right), \\
& e=P_{i-1}\left(q_{1}^{2} q_{2}^{n+2}-q_{2}^{2} q_{1}^{n+2}\right)+P_{n-1}\left(q_{1}^{i+2} q_{2}^{2}-q_{2}^{i+2} q_{1}^{2}\right)+P_{n}\left(q_{1}^{i+2} q_{2}^{3}-q_{2}^{i+2} q_{1}^{3}\right) \\
& +P_{i}\left(q_{1}^{3} q_{2}^{n+2}-q_{2}^{3} q_{1}^{n+2}\right)-\left(P_{i-1} P_{n}-P_{i} P_{n+1}\right)\left(q_{1}^{2} q_{2}^{3}-q_{2}^{2} q_{1}^{3}\right)-\left(q_{1}^{i+2} q_{2}^{n+2}-q_{2}^{i+2} q_{1}^{n+2}\right),
\end{aligned}
$$

and $\mathrm{P}_{\mathrm{i}}$ is the $\mathrm{i}^{\text {th }}$ Pell number.

Proof. We can obtain this conclusion by using (1.5), (1.6), and Theorem 2.4.

The next four theorems are parallel results of type I matrix B and Type II matrix C.

Theorem 2.7. Let $\mathrm{B}$ be given as in (1.2). Then we have

$$
\operatorname{det} B=(-1)^{\frac{n(n-1)}{2}} \sum_{m=1}^{n-1} P_{n-m}\left(\delta_{1} v^{n-2} \epsilon_{m+1}+(-1)^{n+1} \epsilon_{1} v^{n-2} \delta_{m+1}\right),
$$

where $\mathrm{P}_{\mathrm{i}}$ is the $\mathrm{i}^{\text {th }}$ Pell number. 
Proof. Since $\operatorname{det} B=\operatorname{det} \hat{I}_{n} \operatorname{det} A$, where $A$ is a triband Toeplitz matrices with permuted columns as given in (1.1), $\hat{\mathrm{I}}_{n}$ is an inverse unit matrix of order $n$. We obtain this conclusion by using Theorem 2.1 and $\operatorname{det} \hat{\mathrm{I}}_{\mathrm{n}}=(-1)^{\frac{\mathrm{n}(\mathrm{n}-1)}{2}}$.

Corollary 2.8. Let $\delta_{j}=\delta+(n+1-j) d_{1}$ and $\epsilon_{j}=\epsilon+(n+1-j) d_{2}, j=1,2, \ldots, n$ in $(1.2)$, where $\delta, \epsilon, d_{1}$ and $\mathrm{d}_{2}$ are nonzero complex numbers. Then

$$
\begin{aligned}
\operatorname{det} B= & v^{n-2}\left(\delta+n d_{1}\right)\left(\epsilon \frac{P_{n-1}+P_{n}-1}{2}+d_{2} \frac{(n-2) P_{n}+(n-1) P_{n-1}+1}{2}\right) \\
& +(-1)^{n+1} v^{n-2}\left(\epsilon+n d_{2}\right)\left(\delta \frac{P_{n-1}+P_{n}-1}{2}+d_{1} \frac{(n-2) P_{n}+(n-1) P_{n-1}+1}{2}\right),
\end{aligned}
$$

where $\mathrm{P}_{\mathrm{i}}$ is the $\mathrm{i}^{\text {th }}$ Pell number.

Corollary 2.9. Let $\delta_{j}=\delta q_{1}^{j}$ and $\epsilon_{j}=\epsilon q_{2}^{j}, j=1,2, \ldots, n$ in (1.2), where $\delta, \epsilon, q_{1}$ and $q_{2}$ are nonzero complex numbers. Then

$$
\operatorname{det} B=\delta \in q_{1}\left(\frac{q_{2} P_{n}+q_{2}^{2} P_{n+1}-q_{2}^{n+2}}{1+2 q_{2}-q_{2}^{2}}-P_{n} q_{2}\right)-\delta \in q_{2}\left(\frac{q_{1} P_{n}+q_{1}^{2} P_{n+1}-q_{1}^{n+2}}{1+2 q_{1}-q_{1}^{2}}-P_{n} q_{1}\right),
$$

where $\mathrm{P}_{\mathrm{i}}$ is the $\mathrm{i}^{\text {th }}$ Pell number.

Theorem 2.10. Let $\mathrm{B}$ be given as in (1.2). Then we have

$$
B^{-1}=\left(\breve{a}_{i, n+1-j}\right)_{i, j=1}^{n},
$$

where $\breve{\mathrm{a}}_{i, j}$ is the same as (2.6).

Proof. We can obtain this conclusion by using $B^{-1}=A^{-1} \hat{\mathrm{I}}_{n}^{-1}=A^{-1} \hat{\mathrm{I}}_{n}$ and Theorem 2.4, where $A$ is a nonsingular form such as (1.1) given a triband Toeplitz matrices with permuted columns. $\hat{I}_{n}$ is a reverse unit matrix of order $n$.

Corollary 2.11. Let $\delta_{j}=\delta+(n+1-j) d_{1}$ and $\epsilon_{j}=\epsilon+(n+1-j) d_{2}, j=1,2, \ldots, n$ in (1.2), where $\delta, \epsilon, d_{1}$ and $\mathrm{d}_{2}$ are nonzero complex numbers. Then we have

$$
B^{-1}=\left(\dot{a}_{i, n+1-j}\right)_{i, j=1}^{n}
$$

where $\dot{\mathrm{a}}_{\mathrm{i}, \mathrm{j}}$ is the same as (2.12).

Corollary 2.12. Let $\delta_{j}=\delta+(n+1-j) d_{1}$ and $\epsilon_{j}=\epsilon+(n+1-j) d_{2}, j=1,2, \ldots, n$ in (1.2), where $\delta, \epsilon, d_{1}$ and $\mathrm{d}_{2}$ are nonzero complex numbers. Then we have

$$
B^{-1}=\left(\ddot{a}_{i, n+1-j}\right)_{i, j=1}^{n},
$$

where $\ddot{a}_{i, j}$ is the same as (2.13).

Theorem 2.13. Let $\mathrm{C}$ be given as in (1.3). Then we have

$$
\operatorname{det} C=(-1)^{\frac{n(n-1)}{2}} \sum_{m=1}^{n-1} P_{n-m}\left(\delta_{1} v^{n-2} \epsilon_{m+1}+(-1)^{n+1} \epsilon_{1} v^{n-2} \delta_{m+1}\right),
$$

where $\mathrm{P}_{\mathrm{i}}$ is the $\mathrm{i}^{\text {th }}$ Pell number.

Proof. Since $\operatorname{det} C=\operatorname{det} A \operatorname{det} \hat{I}_{n}$, where $A$ is a triband Toeplitz matrices with permuted columns as given in formula (1.1), $\hat{\mathrm{I}}_{n}$ is a reverse unit matrix of order $n$. We obtain this conclusion by using Theorem 2.1 and $\operatorname{det} \hat{I}_{\mathfrak{n}}=(-1)^{\frac{\mathfrak{n}(\mathfrak{n}-1)}{2}}$. 
Corollary 2.14. Let $\delta_{j}=\delta+(n+1-j) d_{1}$ and $\epsilon_{j}=\epsilon+(n+1-j) d_{2}, j=1,2, \ldots, n$ in (1.3), where $\delta, \epsilon, d_{1}$ and $\mathrm{d}_{2}$ are nonzero complex numbers. Then

$$
\begin{aligned}
\operatorname{det} C= & v^{n-2}\left(\delta+n d_{1}\right)\left(\epsilon \frac{P_{n-1}+P_{n}-1}{2}+d_{2} \frac{(n-2) P_{n}+(n-1) P_{n-1}+1}{2}\right) \\
& +(-1)^{n+1} v^{n-2}\left(\epsilon+n d_{2}\right)\left(\delta \frac{P_{n-1}+P_{n}-1}{2}+d_{1} \frac{(n-2) P_{n}+(n-1) P_{n-1}+1}{2}\right),
\end{aligned}
$$

where $\mathrm{P}_{\mathrm{i}}$ is the $\mathrm{i}^{\text {th }}$ Pell number.

Corollary 2.15. Let $\delta_{j}=\delta q_{1}^{j}$ and $\epsilon_{j}=\epsilon q_{2}^{j}, j=1,2, \ldots, n$ in (1.3), where $\delta, \epsilon, q_{1}$ and $q_{2}$ are nonzero complex numbers. Then

$$
\operatorname{det} C=\delta \in q_{1}\left(\frac{q_{2} P_{n}+q_{2}^{2} P_{n+1}-q_{2}^{n+2}}{1+2 q_{2}-q_{2}^{2}}-P_{n} q_{2}\right)-\delta \epsilon q_{2}\left(\frac{q_{1} P_{n}+q_{1}^{2} P_{n+1}-q_{1}^{n+2}}{1+2 q_{1}-q_{1}^{2}}-P_{n} q_{1}\right),
$$

where $\mathrm{P}_{\mathrm{i}}$ is the $\mathrm{i}^{\text {th }}$ Pell number.

Theorem 2.16. Let $\mathrm{C}$ be given as in (1.3). Then we have

$$
C^{-1}=\left(\breve{a}_{n+1-i, j}\right)_{i, j=1}^{n}
$$

where $\breve{\mathrm{a}}_{\mathrm{i}, \mathrm{j}}$ is the same as (2.6).

Proof. We can obtain this conclusion by using $C^{-1}=\hat{\mathrm{I}}_{n}^{-1} A^{-1}=\hat{\mathrm{I}}_{n} A^{-1}$ and Theorem 2.4, where $A$ is a nonsingular form such as (1.1) given a triband Toeplitz matrices with permuted columns. $\hat{I}_{n}$ is a reverse unit matrix of order $n$.

Corollary 2.17. Let $\delta_{j}=\delta+(n+1-j) d_{1}$ and $\epsilon_{j}=\epsilon+(n+1-j) d_{2}, j=1,2, \ldots, n$ in (1.3), where $\delta, \epsilon, d_{1}$ and $\mathrm{d}_{2}$ are nonzero complex numbers. Then we have

$$
\mathrm{C}^{-1}=\left(\dot{\mathrm{a}}_{\mathrm{n}+1-i, j}\right)_{i, j=1}^{\mathrm{n}}
$$

where $\dot{\mathrm{a}}_{\mathrm{i}, \mathrm{j}}$ is the same as (2.12).

Corollary 2.18. Let $\delta_{j}=\delta+(n+1-j) d_{1}$ and $\epsilon_{j}=\epsilon+(n+1-j) d_{2}, j=1,2, \ldots, n$ in (1.3), where $\delta, \epsilon, d_{1}$ and $\mathrm{d}_{2}$ are nonzero complex numbers. Then we have

$$
C^{-1}=\left(\ddot{a}_{n+1-i, j}\right)_{i, j=1}^{n}
$$

where $\ddot{a}_{i, j}$ is the same as (2.13).

\section{Algorithm and numerical example}

In this section, we give an algorithms for finding the determinant and inverse of triband Toeplitz matrices with permuted columns based on Theorems 2.1 and 2.4.

Algorithm 3.1. Calculate the inverse of triband Toeplitz matrices with permuted columns in Theorem 2.4.

Step 1: Input $\delta_{1} \cdots \delta_{n}, v, \epsilon_{1}, \cdots \epsilon_{n}$, order $n$ and generate Pell numbers by (1.4).

Step 2: By the formula (2.5), and output the determinant $\operatorname{det} A$.

Step 3: By the formula (2.6), compute respectively the entries $\breve{a}_{i, j}$.

Step 4: Output the inverse $A^{-1}=\left(\breve{a}_{i, j}\right)_{i, j=1}^{n}$. 
Example 3.2. Consider a $4 \times 4$ triband Toeplitz matrices with permuted columns:

$$
A=\left(\begin{array}{cccc}
1 & 0 & 0 & 4 \\
2 & 1 & 0 & 3 \\
3 & -2 & 1 & 2 \\
4 & -1 & -2 & 1
\end{array}\right)
$$

Show the steps required to calculate the inverse of $A$ in detail according to Algorithm 3.1.

Step 1: Input $\delta_{1}=1, \delta_{2}=2, \delta_{3}=3, \delta_{4}=4, \mathrm{n}=4, v=1, \epsilon_{1}=4, \epsilon_{2}=3, \epsilon_{3}=2, \epsilon_{4}=1$.

Step 2: Based on the formula (2.5), and output the determinant $\operatorname{det} A=-60$.

Step 3: Calculate $\breve{\mathrm{a}}_{1,1}=-\frac{1}{3}, \breve{\mathrm{a}}_{1,2}=\frac{1}{3}, \breve{\mathrm{a}}_{1,3}=\frac{2}{15}, \breve{\mathrm{a}}_{1,4}=\frac{1}{15}, \breve{\mathrm{a}}_{2,1}=-\frac{1}{3}, \breve{\mathrm{a}}_{2,2}=\frac{7}{12}, \breve{\mathrm{a}}_{2,3}=-\frac{1}{6}, \breve{\mathrm{a}}_{2,4}=-\frac{1}{12}, \breve{\mathrm{a}}_{3,1}=$ $-\frac{1}{3}, \breve{\mathrm{a}}_{3,2}=\frac{1}{3}, \breve{\mathrm{a}}_{3,3}=\frac{1}{3}, \breve{\mathrm{a}}_{3,4}=-\frac{1}{3}, \breve{\mathrm{a}}_{4,1}=\frac{1}{3}, \breve{\mathrm{a}}_{4,2}=-\frac{1}{12}, \breve{\mathrm{a}}_{4,3}=-\frac{1}{30}$, and $\breve{\mathrm{a}}_{4,4}=-\frac{1}{60}$.

Step 4: Based on the above step, output $A^{-1}$ as

$$
A^{-1}=\left(\begin{array}{cccc}
-\frac{1}{3} & \frac{1}{3} & \frac{2}{15} & \frac{1}{15} \\
-\frac{1}{3} & \frac{7}{12} & -\frac{1}{6} & -\frac{1}{12} \\
-\frac{1}{3} & \frac{1}{3} & \frac{1}{3} & -\frac{1}{3} \\
\frac{1}{3} & -\frac{1}{12} & -\frac{1}{30} & -\frac{1}{60}
\end{array}\right) .
$$

\section{Acknowledgment}

This research was funded by the Natural Science Foundation of Shandong Province (Grant No. ZR2016AM14) and the PhD Research Foundation of Linyi University (Grant No. LYDX2018BS067), China.

\section{References}

[1] E. L. Allgower, Exact inverses of certain band matrices, Numer. Math., 21 (1973/74), 279-284. 1

[2] E. H. Bareiss, Computational solutions of matrix problems over an integral domain, J. Inst. Math. Appl., 10 (1972), 68-104. 1

[3] M. Bicknell, A primer on the Pell sequence and related sequences, Fibonacci Quart., 13 (1975), 345-349. 1

[4] M. El-Mikkawy, A. Karawia, Inversion of general tridiagonal matrices, Appl. Math. Lett., 19 (2006), 712-720. 1

[5] C. F. Fischer, R. A. Usmani, Properties of some tridiagonal matrices and their application to boundary value problems, SIAM J. Numer. Anal., 6 (1969), 127-142. 1

[6] G. H. Golub, C. F. Van Loan, Matrix Computations, The John Hopkins University Press, Baltimore, (1996). 2

[7] W. D. Hoskins, P. J. Ponzo, Some properties of a class of band matrices, Math. Comp., 26 (1972), 393-400. 1

[8] J. T. Jia, S. M. Li, On the inverse and determinant of general bordered tridiagonal matrices, Comput. Math. Appl., 69 (2015), 503-509. 1

[9] C. M. Jiang, F. F. Zhang, T. X. Li, Synchronization and Antisynchronization of N-coupled Fractional-Order Complex Chaotic Systems with Ring Connection, Math. Methods Appl. Sci., 41 (2018), 2625-2638. 1

[10] T. Koshy, Pell and Pell-Lucas numbers with applications, Springer, New York, (2014). 1

[11] R. P. Mentz, On the inverse of some conariance matrices of Toeplitz type, SIAM J. Appl. Math., 31 (1976), 426-437. 1

[12] T. S. Papatheodorou, Inverses for a class of banded matrices and applications to piecewise cubic approximation, Comput. Appl. Math., 8 (1982), 285-288. 1

[13] L. Rehnqvist, Inversion of certain symmetric band matrices, Nordisk Tidskr. Informationsbehandling (BIT), 12 (1972), 90-98. 1

[14] P. A. Roebuck, S. Barnett, A survey of Toeplitz and releated matrices, Internat. J. Systems Sci., 9 (1978), 921-934. 1

[15] W. F. Trench, Inversion of Toeplitz band matrices, Math. Comp., 28 (1974), 1089-1095. 1

[16] T. Yamamoto, Y. Ikebe, Inversion of band matrices, Linear Algebra Appl., 24 (1979), 105-111. 1

[17] M. Yasar, D. Bozkurt, Another proof of Pell identities by using the determinant of tridiagonal matrix, Appl. Math. Comput., 218 (2012), 6067-6071. 1

[18] Y. Yazlik, N. Taskara, A note on generalized k-Horadam sequence, Comput. Math. Appl., 63 (2012), 36-41.

[19] F. Yilmaz, D. Bozkurt, Hessenberg matrices and the Pell and Perrin numbers, J. Number Theory, 131 (2011), 1390-1396. 1 